\title{
Mechanism of scar formation following Roux-en-Y choledochojejunostomy in a novel rat model of obstructive jaundice
}

\author{
Shao-Cheng Lyu", Jing Wang", Lin Zhou\#, Ji-Qiao Zhu, Fei Pan, Tao Jiang, Ren Lang, Qiang He \\ Department of Hepatobiliary and Pancreaticosplenic Surgery, Beijing Chaoyang Hospital, Capital Medical University, Beijing, China \\ Contributions: (I) Conception and design: SC Lyu, J Wang; (II) Administrative support: R Lang, Q He; (III) Provision of study materials or patients: R \\ Lang, Q He; (IV) Collection and assembly of data: J Wang, L Zhou, JQ Zhu, F Pan, T Jiang; (V) Data analysis and interpretation: J Wang, SC Lyu; (VI) \\ Manuscript writing: All authors; (VII) Final approval of manuscript: All authors. \\ "These authors contributed equally to this work. \\ Correspondence to: Ren Lang; Qiang He. Department of Hepatobiliary and Pancreaticosplenic Surgery, Beijing Chaoyang Hospital, Capital Medical \\ University, No. 8 Gongtinan Road, Chaoyang District, Beijing 100020, China. Email: dr_langren@126.com; heqiang349@sina.com.
}

Background: The present study aimed to analyze the scar formation mechanism following Roux-en-Y choledochojejunostomy (CJS) in a novel rat model of obstructive jaundice.

Methods: The biliary obstruction model of Sprague-Dawley (SD) rats was established in advance, and 24 rats were randomly divided into 4 groups (control group, 1-day ligation group, 3-day ligation group, and 5 -day ligation group). Changes in postoperative weight, common bile duct diameter, and laboratory indexes were analyzed to determine the best operation time. Roux-en-Y CJS in rats was studied based on the model, and the rats were randomly divided into 4 groups [control group, 3-day choledochojejunostomy (CJS) group, 7-day CJS group, and 30-day CJS group]. The same indexes were analyzed, and the characteristics of scar formation were evaluated by histopathology and polymerase chain reaction examination.

Results: The third day after common bile duct ligation is the best time for a Roux-en-Y CJS. The common bile duct diameter expands to $4.2 \mathrm{~mm}$ on average, and these physiological characteristics are consistent with current standard clinical findings. After completing CJS, the rats' weight returned to normal levels, and alanine aminotransferase (ALT), aspartate aminotransferase (AST), total bilirubin (TB), direct bilirubin (DB), and $\mathrm{C}$-reactive protein $(\mathrm{CRP})$ indexes gradually decreased $(\mathrm{P}<0.05)$. Anastomotic stoma diameter tended to narrow with time and was significantly narrower on day 30 than preoperation. After CJS, the expression of $\alpha$-smooth muscle actin ( $\alpha$-SMA) peaked in the early stage and was still higher than that of the control group in the bile duct wall 1 month postoperatively $(\mathrm{P}<0.05)$. Transforming growth factor- $\beta 1$ (TGF- $\beta 1)$ expression gradually increased and was higher than that of the control group at each stage postoperatively $(\mathrm{P}<0.05)$.

Conclusions: The rat Roux-en-Y CJS model is more in line with our surgical model, and the clinical condition has potential applicability for the study of CJS scar formation. Scar formation following CJS in rats is characterized by the activation of fibroblasts caused by early inflammatory stimulation, which leads to the proliferation of collagen and smooth muscle fibers, resulting in scars.

Keywords: Rat model; obstructive jaundice; choledochojejunostomy (CJS); anastomotic stoma; scar formation

Submitted Jul 04, 2020. Accepted for publication Nov 02, 2020.

doi: 10.21037/atm-20-5135

View this article at: http://dx.doi.org/10.21037/atm-20-5135 


\section{Introduction}

Choledochojejunostomy (CJS) is one of the most commonly used basic procedures in biliary surgery and is also a method of biliary reconstruction after resection of pancreatic head carcinoma, ampullary carcinoma, duodenal papillary carcinoma, and other tumors (1-3). In recent years, with increases in the incidence of tumors worldwide (4), the use of CJS in the clinical setting has also increased. Choledoenterostomy has evolved into cholecystojejunostomy, choledochoduodenostomy, and CJS (5). Until recently, Roux-en-Y CJS has been the most commonly used CJS in the clinical setting. However, with the promotion and popularization of this technique, the follow-up problems associated with this procedure are becoming more apparent. Because $t$ valve function in the sphincter of Oddi is inevitably depreciated in CJS, the bile duct is directly connected to the intestinal tract, which leads to the inevitable occurrence of bile reflux after surgery. Once the intestinal contents flow into the biliary tract, there is a risk of cholangitis; repeated cholangitis stimulation can cause scar tissue hyperplasia and eventually lead to CJS stricture and even cancer $(6,7)$. According to the previously published studies, the anastomosis's stenosis rate is as high as $13-58 \% 1$ year after CJS (8-11). Once the anastomotic scar is formed, the effect of drug treatment will be limited, and reoperation or interventional stenting is the only effective treatment option (12). However, there is still a risk of anastomotic restenosis after retreatment. Patients with repeated biliary tract operations due to anastomotic stricture are often encountered in clinical practice. Therefore, the study of scar formation characteristics in CJS is of significance for preventing and treating CJS strictures. Nevertheless, there is still a lack of animal models of CJS suitable for basic research. In the present study, the rat Roux-en-Y CJS model was established for the first time further to analyze the characteristics of the scar formation of choledochojejunal anastomosis.

We present the following article following the ARRIVE reporting checklist (http://dx.doi.org/10.21037/atm-20$5135)$.

\section{Methods}

\section{Ethics approval}

The study was performed under a project license (No. 2019-D.-304) granted by the Ethics Committee of Beijing Chaoyang Hospital and complied with the institutional guidelines for the care and use of animals.

\section{Experimental animals}

Specific Pathogen Free (SPF)-grade male Sprague-Dawley (SD) rats aged 6-8 weeks and weighing about $250 \mathrm{~g}$ were selected for the present study. The experimental animals were purchased from Beijing Weitong Lihua Animal Experimental (Beijing, China), and were housed at the Medical Research Center of Beijing Chaoyang Hospital (Beijing, China) at normal room temperature with standard chow. The circadian rhythm of the rats was monitored for $12 \mathrm{~h}$.

\section{Perioperative management}

The rats fasted (including no access to water) for $6 \mathrm{~h}$ before surgery. Surgical anesthesia was performed by an intraperitoneal injection of chloral hydrate $(0.5 \mathrm{~mL} / 100 \mathrm{~g})$. After completing the operation, the rats were put into a $37^{\circ}$ rewarming table to recover from anesthesia. The rats were not given water for another $12 \mathrm{~h}$ after the operation, and normal feeding was resumed after $12 \mathrm{~h}$. All the rats were killed by inferior vena cava bloodletting after the tissue was obtained by laparotomy. All operations were in line with the ethical principles of laboratory animal welfare and approved by the Ethical member Association of Beijing Chaoyang Hospital affiliated to Capital Medical University.

\section{Surgical technique}

\section{Establishment of biliary obstruction model in rats}

Twenty-four SD rats were randomly divided into the control group, 1-day ligation group (biliary duct ligation for the 1-day group), 3-day ligation group (biliary duct ligation for the 3-day group), and 5-day ligation group (biliary duct ligation for the 5 -day group) ( $\mathrm{n}=6 /$ group). All rats were weighed before the operation. Rats in the control group were killed directly after the common bile duct diameter was measured with a Vernier caliper, and rats in the ligation group were treated with common bile duct ligation (Figure 1). An abdominal incision was made to access the common bile duct measured with the Vernier caliper. The common bile duct was ligated twice with 7-0 silk thread in the middle and lower parts of the common bile duct. Finally, the abdomen was closed continuously with 4-0 silk thread. The rats were killed at the corresponding time point, reweighed at the time of death, and the dilated 

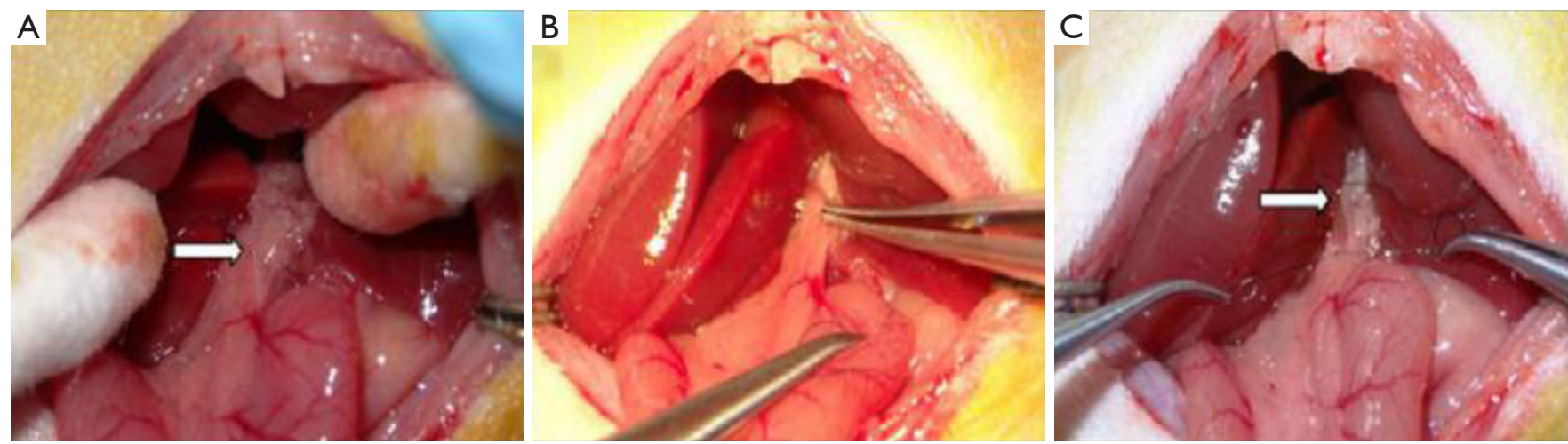

Figure 1 Common bile duct ligation. (A) Explore and expose the common bile duct (the shape of the common bile duct can be seen at the arrow indication). (B) Properly separate the posterior wall of common bile duct and prepare for ligation. (C) Ligation of the common bile duct with 7-0 silk thread (slight dilatation of the proximal common bile duct can be seen at the arrow indication).

common bile duct diameter was measured with a Vernier caliper. In total, $4 \mathrm{~mL}$ of blood was collected from the inferior vena cava for follow-up after the rats were killed.

\section{Roux-en-Y CJS}

Twenty-four SD rats were randomly divided into the control group, 3-day CJS group (CJS for the 3-day group), 7-day CJS group (CJS for the 7-day group), and 30-day CJS group (CJS for the 30-day group) (n=6/group). All rats were weighed before the operation. Six rats in the control group were only treated with common bile duct ligation. The best operation time was again determined in the first part of the experiment. The common bile duct diameter was measured with a Vernier caliper, and the rats were killed directly after the common bile duct tissue was obtained. Eighteen rats in the experimental group underwent reoperation at the best operation time, as determined in the first part of the experiment, and Roux-en-Y CJS was performed (Figure 2). The common bile duct was exposed and dilated through the original surgical incision, and the common bile duct was severed at the upper end of the ligation line. The jejunum was severed at a distance of $3 \mathrm{~cm}$ from the distal ligament of Treitz, and the broken end of the distal jejunum was sutured with a 6-0 Prolene suture; the jejunum wall was opened according to the diameter of the common bile duct at a distance of $1 \mathrm{~cm}$ from the broken end. CJS was performed by end-to-side anastomosis of the distal choledochal jejunal mucosa, continuous anastomosis of an 8-0 Prolene line in the posterior wall, and intermittent anastomosis of an 8-0 Prolene line in the anterior wall. The jejunal wall was opened at a distance of $4 \mathrm{~cm}$ from the anastomotic stoma, and the 6-0 Prolene line was used to complete the endto-side anastomosis between the proximal jejunal stump and the distal jejunal lateral wall. After confirming no bleeding and biliary fistula, the abdomen was closed with 4-0 silk thread. The rats were killed at the corresponding time point, reweighed at the time of death, and the dilated common bile duct diameter was measured with a Vernier caliper. In total, $4 \mathrm{~mL}$ blood was collected from the inferior vena cava; anastomotic tissue of the common bile duct and jejunum were obtained for follow-up after the rats were killed.

\section{Laboratory examination}

The blood samples obtained from the rats were placed at room temperature for $30 \mathrm{~min}$ and then separated by centrifugation $(1,125 \mathrm{~g} / \mathrm{min}$ for $10 \mathrm{~min})$ to obtain serum. Alanine aminotransferase (ALT), aspartate aminotransferase (AST), total bilirubin (TB), direct bilirubin (DB), and C-reactive protein (CRP) were determined by enzymelinked immunosorbent assay.

\section{Histological examination}

The common bile duct or CJS anastomotic stoma tissue obtained from rats was divided into 2 pieces. The first piece (4- $\mu \mathrm{m}$ section) was fixed in $10 \%$ formalin for $48 \mathrm{~h}$, and the tissue specimen was embedded in wax after dehydration, followed by hematoxylin-eosin (HE) staining, Masson staining, $\alpha$-smooth muscle actin ( $\alpha$-SMA) immunohistochemical staining, transforming growth 

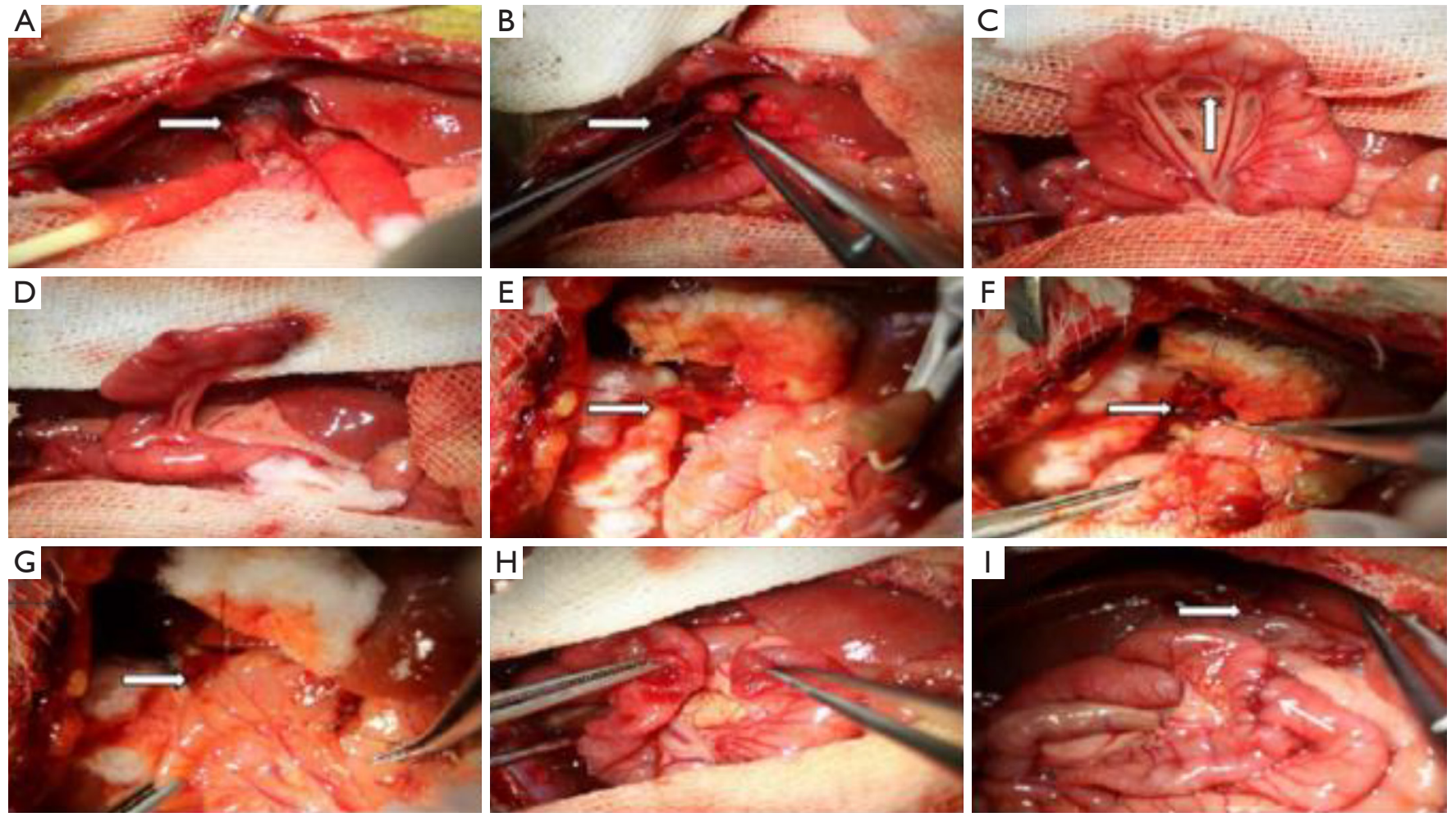

Figure 2 Roux-en-Y choledochojejunostomy. (A) Expose dilated common bile duct (obvious dilatation of common bile duct can be seen at the arrow indication). (B) Severed common bile duct (common bile duct diameter $4.3 \mathrm{~mm}$ can be seen at the arrow indication). (C) The jejunum was severed at the distal $3 \mathrm{~cm}$ of the Treitz ligament. (D) Closure of distal jejunum with 6-0 prolene suture. (E) Common bile duct lumen exposed on both sides of common bile duct suspended by 8-0 prolene line. (F) Continuous suture of posterior wall of common bile duct and jejunum with 8-0 prolene line. (G) Intermittent suture of the anterior wall of common bile duct and jejunum with 8-0 prolene line. (H) End-to-side jejunojejunostomy with 6-0 prolene line. (I) Operation completion diagram (thick arrow indicates choledochojejunostomy, thin arrow indicates enterostomy).

factor- $\beta 1$ (TGF- $\beta 1$ ) immunohistochemical staining, and observation. The second specimen was immediately transferred to liquid nitrogen for preservation and the relative quantitative detection of $\alpha$-SMA and TGF- $\beta 1$ by reverse transcription-polymerase chain reaction (RT-PCR). Total RNA was extracted by TRIzol method (Invitrogen, USA), and cDNA synthesis was carried out by using SuperScript First Chain Synthesis System (Thermo, USA). The primers were synthesized by raw engineering, and realtime PCR was carried out using SYBR Green PCR Master Mix (Roche, Switzerland).

Primer information was as follows: GAPDH primer: forward: 5'-GGCAAGTTCAACGGCACAG-3' and reverse: 5'-CGCCAGTAGACTCCACGACA-3'; $\alpha$-SMA primer: forward: 5'-ATGCTTCTGGACGTACAACTG-3' and reverse: 5'-GGAATAGCCACGCTCAGTCAG-3';
TGF- $\beta 1$ primer: forward: 5'-ATAGCAACAATTCCTGG CGTTACCTT-3' and reverse: 5'-CCTGTATTCCGTCT CCTTGGTTCAG-3'.

\section{Statistical analysis}

Measurement data are expressed by mean \pm standard deviation, following a normal distribution, and median (quartile spacing) in non-normal distribution. Comparing the measurement data between the 2 groups, the $t$-test was used for the normal distribution, and the rank-sum test was used for the non-normal distribution. Error diagram was used to describe the observation index. Differences were considered statistically significant when $\mathrm{P}<0.05$, and all data were analyzed by SPSS version 22.0 software (IBM, Armonk, NY, USA). 
Table 1 Changes of various indexes of rats after common bile duct ligation

\begin{tabular}{|c|c|c|c|c|}
\hline Item & Control group & 1-day ligation group & 3-day ligation group & 5-day ligation group \\
\hline Diameter of bile duct $(\mathrm{mm})$ & $1.2 \pm 0.2$ & $1.3 \pm 0.1$ & $4.5 \pm 0.5^{\mathrm{ab}}$ & $7.8 \pm 1.0^{\mathrm{abc}}$ \\
\hline $\operatorname{ALT}(\mathrm{U} / \mathrm{L})$ & $6.8 \pm 1.5$ & $27.7 \pm 6.0^{a}$ & $90.2 \pm 8.4^{\mathrm{ab}}$ & $103.7 \pm 10.1^{\mathrm{abc}}$ \\
\hline AST (U/L) & $10.7 \pm 1.2$ & $24.0 \pm 3.2^{\mathrm{a}}$ & $58.7 \pm 9.8^{\mathrm{ab}}$ & $66.0 \pm 10.9^{\mathrm{ab}}$ \\
\hline DB (umol/L) & $15.2 \pm 2.6$ & $46.2 \pm 12.8^{\mathrm{a}}$ & $91.8 \pm 8.3^{\mathrm{ab}}$ & $98.3 \pm 9.9^{\mathrm{ab}}$ \\
\hline $\mathrm{CRP}(\mathrm{mg} / \mathrm{L})$ & $2.4 \pm 0.2$ & $2.9 \pm 0.3^{\mathrm{a}}$ & $2.8 \pm 0.2^{\mathrm{a}}$ & $2.5 \pm 0.2^{b c}$ \\
\hline
\end{tabular}

${ }^{a}$ refers to the comparison with the control group $(P<0.05) ;{ }^{b}$ refers to the comparison with the 1-day ligation group $(P<0.05) ;{ }^{c}$ refers to the comparison with the 3 -day ligation group $(P<0.05)$. ALT, alanine aminotransferase; AST, aspartate aminotransferase; TB, total bilirubin; $D B$, direct bilirubin; CRP, C-reactive protein.
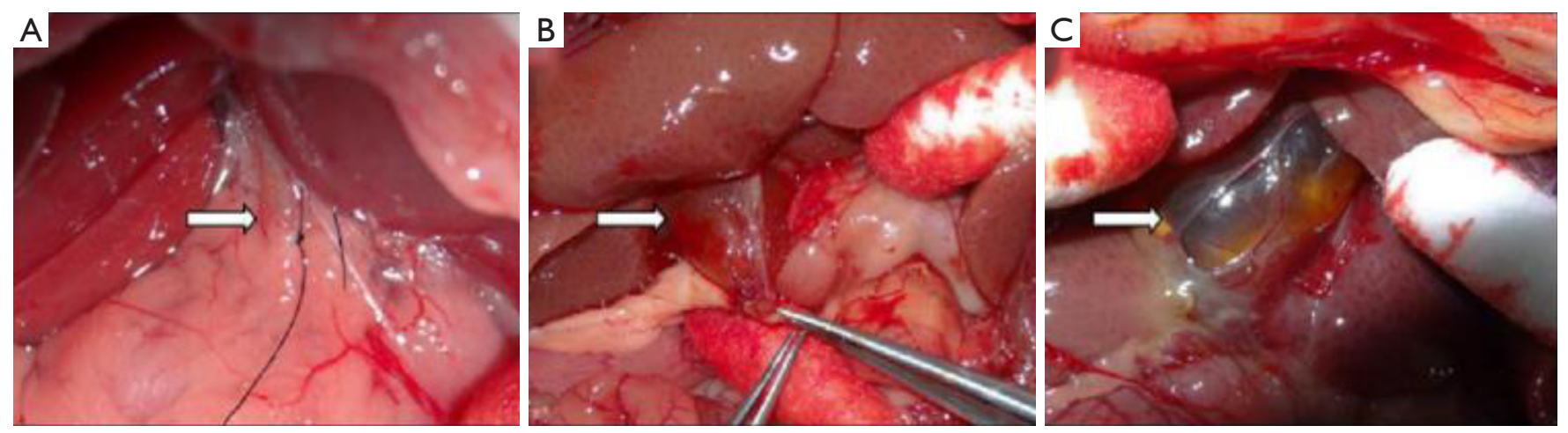

Figure 3 Changes of common bile duct diameter after common bile duct ligation. (A) In the 1-day ligation group, the dilatation of bile duct was not obvious (at the arrow, $1.3 \mathrm{~mm}$ ). (B) In the 3-day ligation group, the common bile duct was obviously dilated (at the arrow, $4.6 \mathrm{~mm}$ ). (C) In the 5-day ligation group, the common bile duct was obviously dilated into a transparent shape (at the arrow, $7.6 \mathrm{~mm}$ ).

\section{Results}

\section{Indexes of rats after common bile duct ligation}

The average operation time for common bile duct ligation was $23.4 \pm 3.8 \mathrm{~min}$. There were no intraoperative rat deaths. The success rate of modeling was $100 \%$. Changes in body weight, common bile duct diameter, and rats' laboratory indexes at different time points after common bile duct ligation are shown in Table 1 and Figures 3 and 4. With the extension of ligation time, the rats' weight gradually decreased, the diameter of the common bile duct gradually widened, and the ALT, AST, TB, and DB gradually increased $(\mathrm{P}<0.05)$. On the first day after ligation, the common bile duct was not dilated, and the CJS was unable to be completed. On the third day after ligation, the common bile duct's average diameter was $4.2 \mathrm{~mm}$, and the follow-up operation could be safely performed. Although the common bile duct diameter was the widest on the fifth day after ligation, the follow-up anastomosis could not be completed due to the common bile duct's thin and fragile wall. Therefore, we believe that the third day after primary choledochal ligation is the best time for Roux-en-Y CJS.

\section{Indexes of rats after Roux-en-Y CFS}

The average operation time of Roux-en-Y CJS was 56.4 \pm $9.6 \mathrm{~min}$. There were no intraoperative rat deaths. Two rats died on the second and third days after the operation, respectively, and the biliary fistula was found by autopsy. The success rate of modeling was $89 \%$. Changes in weight, the diameter of the anastomotic stoma, and laboratory indexes in rats at different time points after Roux-en-Y 



Figure 4 Changes of weight, common bile duct diameter and laboratory indexes of rats at different time points after common bile duct ligation (* refers to the comparison with the control group, $\mathrm{P}<0.05)$.

Table 2 Changes of various indexes of rats after Roux-en-Y choledochojejunostomy

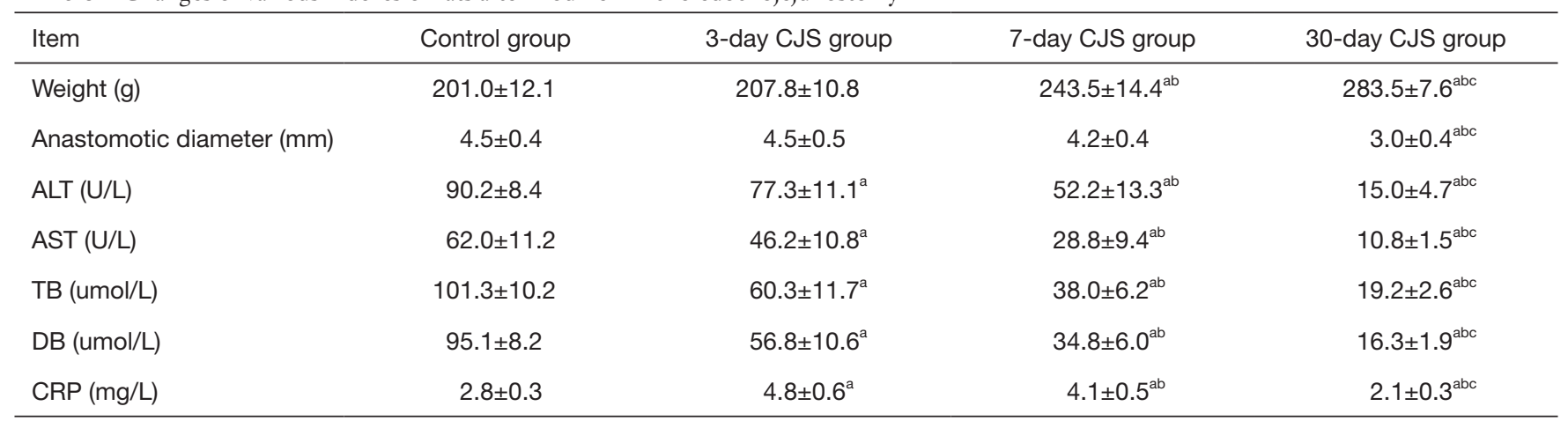

\footnotetext{
${ }^{a}$ refers to the comparison with the control group $(\mathrm{P}<0.05) ;{ }^{b}$ refers to the comparison with the 3 -day $\mathrm{CJS}$ group $(\mathrm{P}<0.05) ;{ }^{\circ}$ refers to the comparison with the 7-day CJS group $(P<0.05)$. ALT, alanine aminotransferase; AST, aspartate aminotransferase; TB, total bilirubin; DB, direct bilirubin; CRP, C-reactive protein; CJS, choledochojejunostomy.
}

CJS are shown in Table 2, Figures 5 and 6. The rats' weight gradually recovered and returned to preoperative level after about 1 week and increased significantly at 30 days after the operation $(\mathrm{P}<0.05)$. ALT, AST, TB, DB, and CRP indexes gradually decreased $(\mathrm{P}<0.05)$ and returned to normal levels 30 days after the operation. The anastomotic stoma diameter was narrowed with time and was significantly narrower 30 days after the operation than before. 

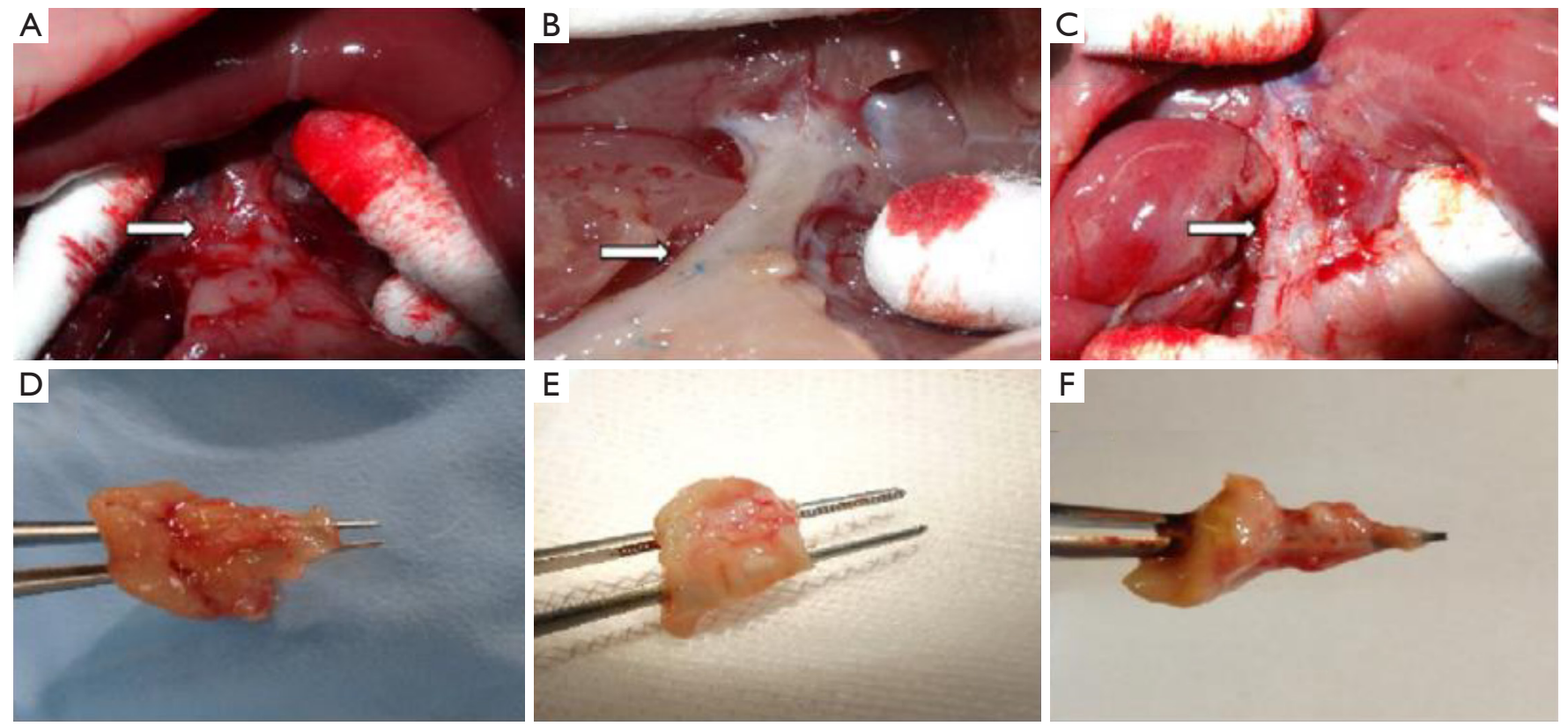

Figure 5 Changes of the anastomotic stoma diameter after Roux-en-Y choledochojejunostomy (CJS). (A) In the 3-day CJS group, no significant change in the diameter of anastomotic stoma (at the arrow, $4.3 \mathrm{~mm}$ ), and the wall of common bile duct was transparent. (B) In the 7-day CJS group, no obvious change in the diameter of anastomotic stoma (at the arrow, $4.2 \mathrm{~mm}$ ), and the white scar was formed in the wall of common bile duct. (C) In the 30-day CJS group, the diameter of anastomotic stoma was obviously narrow (at the arrow, $3.2 \mathrm{~mm}$ ), and the wall of common bile duct shows scar like changes. (D) Anastomotic tissue on the $3^{\text {rd }}$ day after CJS. (E) Anastomotic tissue on the $7^{\text {th }}$ day after CJS. (F) Anastomotic tissue on the $30^{\text {th }}$ day after CJS.
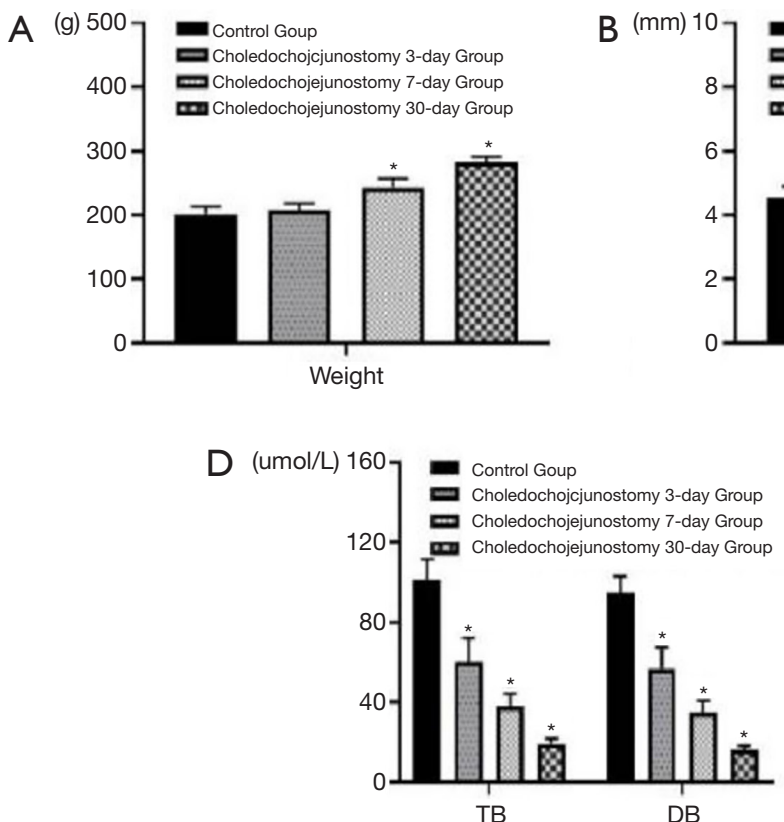

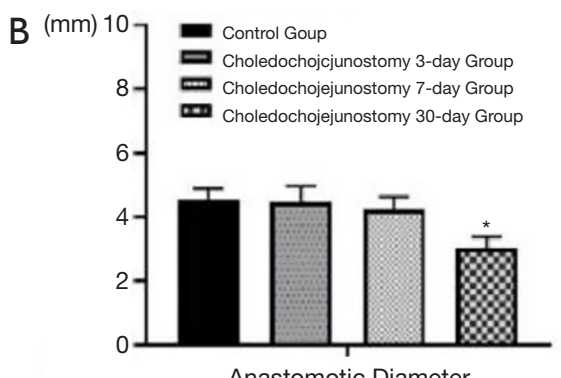

Anastomotic Diameter


Figure 6 Changes of weight, common bile duct diameter and laboratory indexes in rats at different time points after Roux-en-Y choledochojejunostomy (* refers to the comparison with the control group, $\mathrm{P}<0.05$ ). 

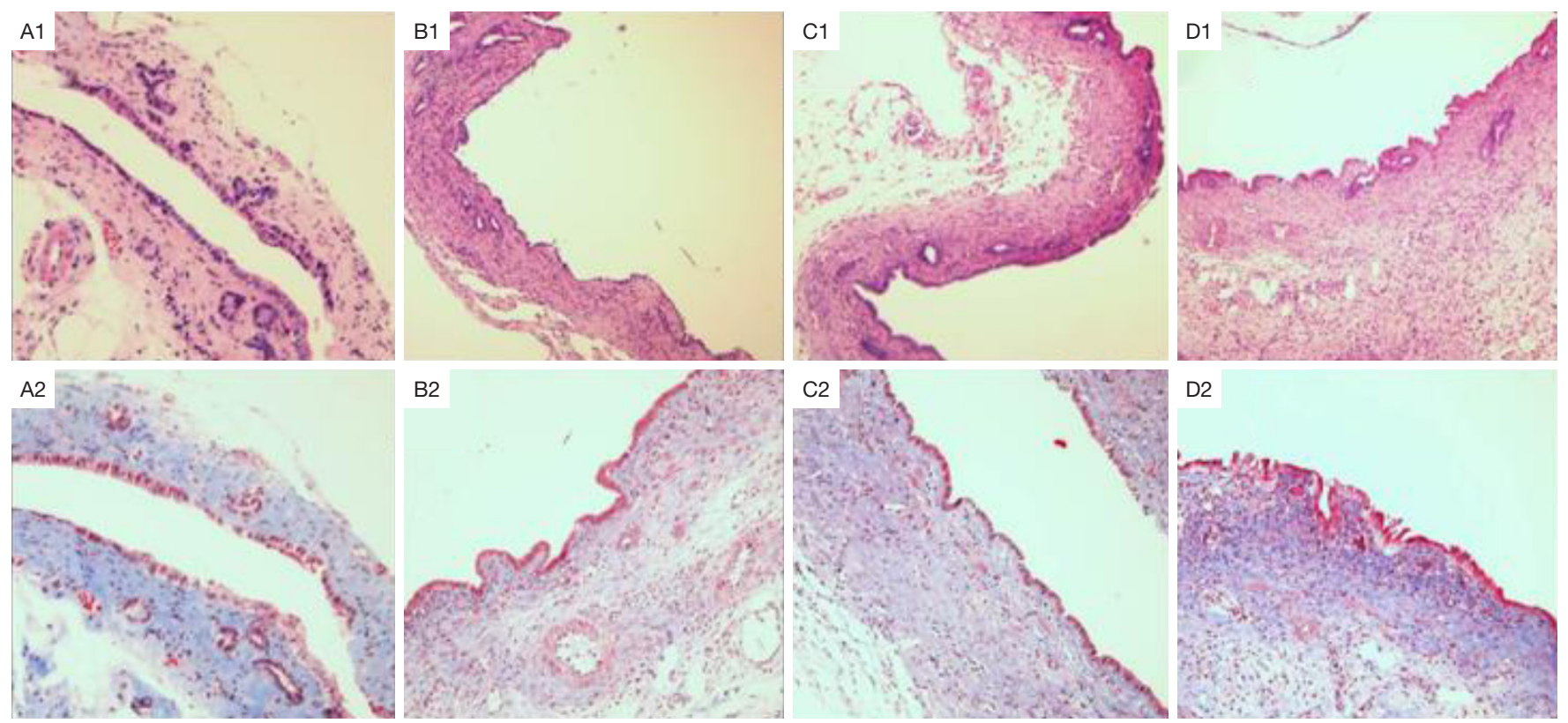

Figure $7 \mathrm{HE} \&$ Masson staining of anastomotic stoma after Roux-en-Y CJS (×200). (A1) HE staining in the control group. (A2) Masson staining in the control group. (B1) HE staining in the 3-day CJS group. (B2) Masson staining in the 3-day CJS group. (C1) HE staining in the 7-day CJS group. (C2) Masson staining in the 7-day CJS group. (D1) HE staining in the 30-day CJS group. (D2) Masson staining in the 30-day CJS group. CJS, choledochojejunostomy.

\section{The pathological condition of choledochojejunal anastomotic stoma}

The results of HE staining, Masson staining, $\alpha$-SMA immunohistochemical staining, and TGF- $\beta 1$ immunohistochemical staining in the anastomosis of rats at different points after surgery are shown in Figures 7 and 8 . The rats' normal bile duct wall was divided into 3 layers: mucosa, the muscle layer, and adventitia. The bile duct epithelium was formed with a monolayer columnar epithelium. HE staining showed that, in the control group, the endothelium of the bile duct was arranged closely, and infiltration of inflammatory cells was rare. In the 3-day CJS group, the bile duct endothelium structure was still intact, and inflammatory cells' infiltration could be seen under the mucosa. In the 7-day CJS group, the bile duct epithelium structure was still intact, some small blood vessels proliferated, and inflammatory cell infiltration was aggravated. In the 30-day CJS group, the bile duct epithelium structure was still intact, and the bile duct wall was thickened with a large number of inflammatory cell infiltration. Masson staining showed, in the control group, uniform light blue staining and that the collagen fibers were neatly arranged. In the 3-day
CJS group, collagen fibers' arrangement in the bile duct wall was irregular, and a small amount of red-stained smooth muscle fibers appeared. In the 7-day CJS group, the blue staining of collagen fibers in the bile duct wall deepened, and some of the fibers were broken. In the 30-day CJS group, the collagen fibers were disorderly in the bile duct wall, and some red-stained smooth muscle fibers could be seen. $\alpha$-SMA staining showed yellow staining of vascular smooth muscle cells around the bile duct wall in the control group; some yellowstained smooth muscle cells could be seen in the wall of small vessels. Many yellow-stained smooth muscle cells could be seen in the bile duct wall and were arranged regularly in the 3-day CJS group and the 7-day CJS group; many yellow-stained smooth muscle cells could be seen in the bile duct wall and were disorderly. In the 30-day CJS group, uniformly arranged yellow smooth muscle cells could be seen in the whole layer of the bile duct wall, and TGF- $\beta 1$ staining was negative. After CJS, inflammatory cells' infiltration in the bile duct wall of the anastomotic stoma gradually aggravated with time. The collagen and smooth muscle fibers proliferated obviously, and the expression of $\alpha$-SMA and TGF- $\beta 1$ in the bile duct wall gradually increased. 

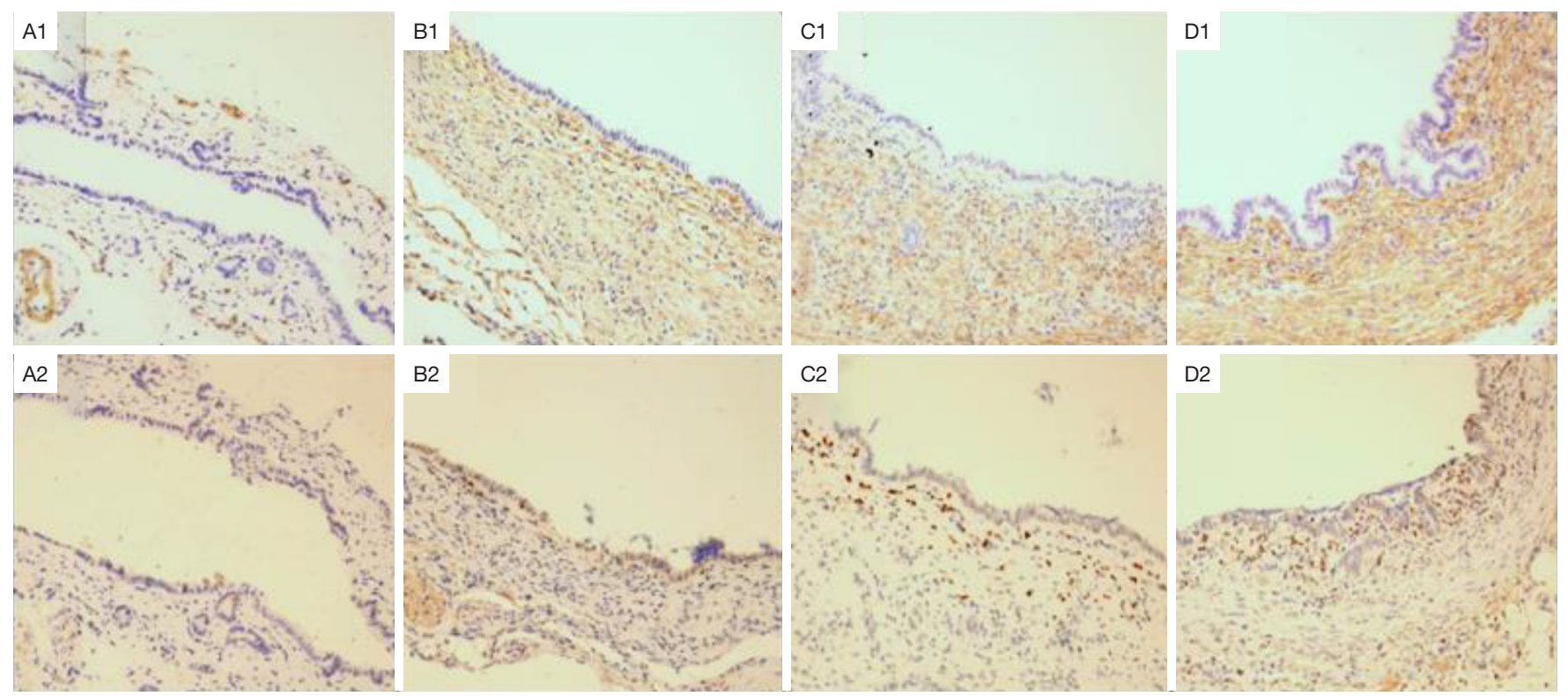

Figure 8 Immunohistochemical staining of $\alpha$-SMA \& TGF- $\beta 1$ in the anastomotic stoma after Roux-en-Y CJS ( $\times 200)$. (A1) IHC staining of $\alpha$-SMA in the control group. (A2) IHC staining of TGF- $\beta 1$ in the control group. (B1) IHC staining of $\alpha$-SMA in the 3-day CJS group. (B2) IHC staining of TGF- $\beta 1$ in the 3 -day CJS group. (C1) IHC staining of $\alpha$-SMA in the 7 -day CJS group. (C2) IHC staining of TGF- $\beta 1$ in the 7-day CJS group. (D1) IHC staining of $\alpha$-SMA in the 30 -day CJS group. (D2) IHC staining of TGF- $\beta 1$ in the 30 -day CJS group. CJS, choledochojejunostomy.

\section{Relative quantitative detection of PCR in choledochojejunal anastomotic stoma}

The changes in the RT-PCR relative quantitative detection of $\alpha$-SMA and TGF- $\beta 1$ in rat anastomotic stoma tissue at different time points after CJS are shown in Table 3 and Figure 9. After CJS, the expression of $\alpha$-SMA peaked in the early stage, then decreased gradually; its expression was still higher than that in the control group at 1 month postoperation $(\mathrm{P}<0.05)$. The expression of TGF- $\beta 1$ gradually increased and was higher than that in the control group at each stage after surgery $(\mathrm{P}<0.05)$.

\section{Discussion}

The reconstruction of the bile outflow tract is a basic technique in biliary surgery $(13,14)$. In 1887 , Monastyski first reported cholecystojejunostomy, and the following year, Riedl pioneered choledochoduodenostomy. In 1893, César Roux designed a Roux-en-Y jejunal loop for gastrojejunostomy, later used in CJS to prevent intestinal fluid reflux (15). At present, Roux-en-Y CJS is the most commonly used method for bile duct reconstruction in the clinical setting $(16,17)$. However, regardless of the type of
CJS, because of the deprecation of the sphincter of Oddi, the problem of intestinal fluid reflux cannot be solved completely, and repeated cholangitis stimulation will cause scar tissue hyperplasia and eventually lead to anastomotic stricture, and even cancer (18-20). Therefore, determining the characteristics of scar formation in CJS is of significance for the prevention and treatment of CJS strictures.

Previous literature has indicated that most animal models used in CJS include large animals, such as rabbits, dogs, and pigs (21-24). Experiments with large animals are expensive, and there are problems associated with feeding and managing large animals. de Aro Braz et al. and Corbi et al. previously established a rat model of CJS to study the effect of related indexes of biliary cirrhosis after removal of biliary obstruction. However, the specific method of CJS was common bile duct duodenal anastomosis $(25,26)$. However, at present, the anastomosis used in clinical work is Roux-en-Y CJS. To make the rat model more in line with the clinical characteristics, we improved based on the previous model and adopted the Roux-en-Y CJS. The Roux-en-Y CJS model in rats designed in the present study has the following advantages. First, it is a method of 2 -stage operation; bile duct dilatation is caused by the model 
Table 3 Changes of $\alpha$-SMA and TGF- $\beta 1$ expression in anastomotic stoma after Roux-en-Y choledochojejunostomy

\begin{tabular}{lcccc}
\hline Item & Control group & 3-day CJS group & 7-day CJS group & 30-day CJS group \\
\hline$\alpha$-SMA & $0.3 \pm 0.2$ & $9.5 \pm 2.2^{\mathrm{a}}$ & $8.0 \pm 3.2^{\mathrm{a}}$ & $3.1 \pm 0.9^{\mathrm{abc}}$ \\
TGF- $\beta 1$ & $0.4 \pm 0.2$ & $1.9 \pm 0.6^{\mathrm{a}}$ & $2.0 \pm 0.8^{\mathrm{a}}$ & $3.2 \pm 0.7^{\mathrm{abc}}$ \\
\hline
\end{tabular}

${ }^{a}$ refers to the comparison with the control group $(\mathrm{P}<0.05) ;{ }^{b}$ refers to the comparison with the 3 -day CJS group $(\mathrm{P}<0.05) ;{ }^{\mathrm{c}}$ refers to the comparison with the 7 -day CJS group $(\mathrm{P}<0.05)$. $\alpha$-SMA, $\alpha$-smooth muscle actin; TGF- $\beta 1$, transforming growth factor- $\beta 1$; CJS, choledochojejunostomy.



Figure 9 Changes of $\alpha$-SMA \& TGF- $\beta 1$ expression in the anastomotic stoma at different time points after Roux-en-Y choledochojejunostomy ( ${ }^{*}$ refers to the comparison with the control group, $\mathrm{P}<0.05)$.

of primary obstruction, which is more in line with the characteristics of clinical patients. Second, Roux-en-Y CJS is adopted, which is more in line with the characteristics of clinical surgery. Third, rats' feeding and management is relatively simple and does not require professional animal anesthesiologists and keepers' cooperation. Fourth, the diameter of the bile duct in normal rats is about $1-2 \mathrm{~mm}$, and can dilate to about $5 \mathrm{~mm}$ after 3 days of ligation, which can fully meet the needs of CJS. Fifth, CJS follows the clinical method of the continuous posterior wall and the intermittent anterior wall (27), and with practice, we can skillfully master the technique of CJS in rats. Finally, rats' metabolism is fast, the feeding cycle is short, and the scar formation of CJS can be observed 1 month after the operation.

In the present study, we studied the physiological changes of the common bile duct in rats after ligation. We found that after ligation of the common bile duct, rats showed typical symptoms of obstructive jaundice, yellowing of hair and urine, weight loss, and dilatation of the common bile duct, with the prolongation of ligation time. Laboratory tests indicated that ALT, AST, TB, and
DB indexes were significantly increased. We also found that on the third day after common bile duct ligation, the common bile duct diameter was dilated to 3-5 times, body weight was reduced by $20 \%$, and the bilirubin increased by 5-8 times. Compared with other time points, the third day after common bile duct ligation is the best time point for Roux-en-Y CJS. We further investigated the physiological changes after CJS in rats and observe that, after CJS, biliary obstruction improved rapidly, body weight returned to normal levels 1 week after surgery. The ALT, AST, TB, and DB indexes returned to normal levels 1 month after the operation. However, the diameter of the anastomotic stoma in rats gradually developed cicatricial coarctation with time.

Pathophysiological change of scar formation is an extremely complex process and involves multi-tissue, multicell, multi-factor, and multi-component participation. It can be roughly divided into the inflammatory phase, cell proliferation phase, and tissue remodeling phase, and inflammatory reaction is the initial link of scar formation (28). After CJS, the anastomotic stoma was found to have repeated inflammatory reactions due to intestinal reflux. $\alpha$-SMA was the most prominent marker for the development of myofibroblasts into hypertrophic scars and was mainly found in myofibroblasts $(29,30)$. There were no myofibroblasts in the normal bile duct wall and only a few smooth muscle cells in the small vessels around it. Myofibroblasts are cells with characteristics of both fibroblasts and smooth muscle cells, mainly transformed from fibroblasts due to local stimulation. Zhang et al. found that, in the early stage of the healing process, $\alpha$-SMA-positive cells appeared in all layers of the bile duct wall after end-to-end anastomosis of the common bile duct in guinea pigs, mainly concentrated in the muscular and intimal layers, and myofibroblasts proliferated obviously at the same time; however, at 6 months after the operation, the number of $\alpha$-SMApositive cells decreased (31). We also found that in the early stage after CJS, $\alpha$-SMA cells were highly expressed, 
which reflected that the anastomotic stoma was stimulated by inflammation; fibroblasts proliferated actively and gradually transformed into myofibroblasts. With scar hyperplasia's stability, the expression of $\alpha$-SMA decreased gradually, and the pathological staining of the common bile duct wall showed that $\alpha$-SMA staining gradually deepened with time.

TGF- $\beta 1$ is known to be the most closely related cytokine to scar formation in the body. A variety of cells can secrete it, especially activated inflammatory cells (32). TGF- $\beta 1$ can act on fibroblasts through paracrine, autocrine, and other biologic pathways to participate in the process of wound repair $(33,34)$. It can directly stimulate fibroblasts, promote their proliferation, and then convert them into myofibroblasts to form scars; however, it can also activate fibroblasts originally in the quiescent stage and promote the formation of the collagen matrix. It plays an important role in inhibiting the collagen matrix decomposition, resulting in scars' continuous accumulation. TGF- $\beta 1$ also plays an important role in linking inflammation, injury, and scar of tissues and organs $(35,36)$, providing the necessary signals for the whole healing process of tissues and organs. Once the expression of these signals is abnormal, it will lead to poor healing and scar formation. Siqueira et al. showed that the proportion of collagen and TGF- $\beta 1$ in the common bile duct wall of pigs after end-to-end anastomosis was significantly higher than those in the control group (37). In our research, we also found that TGF- $\beta 1$ was highly expressed in the early stage after CJS, and the expression increased gradually with time. Pathological staining of the common bile duct wall showed that the yellow staining granules of TGF- $\beta 1$ staining gradually increased with time.

During the process of tissue healing, once fibroblasts begin to proliferate widely and even reach the stage of tissue remodeling, it is difficult to regulate and block the formation of scar tissue (38). Through Masson staining, we found a large number of collagen fibers and smooth muscle fibers in the anastomotic stoma 1 month after the operation, and the fibers were disorderly and broken. The characteristics of scar formation in CJS are as follows (39-41): inflammatory cell infiltration and fibroblast activation appeared in the early stage of anastomotic stoma healing; with time, infiltration of inflammatory cells in the anastomotic stoma was gradually aggravated, fibroblasts and myofibroblasts proliferated, and collagen fibers and smooth muscle fibers increased significantly, and eventually led to the proliferation of granulation tissue and scar formation. If an early intervention can reduce the anastomosis's inflammatory reaction and the activation of fibroblasts, it could prevent CJS scars' formation. Therefore, the rat Roux-en-Y CJS model established in the present study provides a good basis for follow-up research.

\section{Conclusions}

The rat Roux-en-Y CJS model is in line with the clinical condition and has a good application for the study of CJS scar formation. Scar formation following CJS in rats is characterized by the activation of fibroblasts caused by early inflammatory stimulation, which leads to the proliferation of collagen and smooth muscle fibers, finally forming a scar.

\section{Acknowledgments}

Funding: The present study was supported by the Beijing Natural Science Foundation (Grant No. 7194274).

\section{Footnote}

Reporting Checklist: The authors have completed the ARRIVE reporting Checklist. Available at http://dx.doi. org/10.21037/atm-20-5135

Data Sharing Statement: Available at http://dx.doi. org/10.21037/atm-20-5135

Conflicts of Interest: All authors have completed the ICMJE uniform disclosure form (available at: http://dx.doi. org/10.21037/atm-20-5135). The authors have no conflicts of interest to declare.

Ethical Statement: The authors are accountable for all aspects of the work in ensuring that questions related to the accuracy or integrity of any part of the work are appropriately investigated and resolved. The study was performed under a project license (No. 2019-D.-304) granted by the Ethics Committee of Beijing Chaoyang Hospital and complied with institutional guidelines for animals' care and use.

Open Access Statement: This is an Open Access article distributed in accordance with the Creative Commons Attribution-NonCommercial-NoDerivs 4.0 International License (CC BY-NC-ND 4.0), which permits the noncommercial replication and distribution of the article with the strict proviso that no changes or edits are made and the 
original work is properly cited (including links to both the formal publication through the relevant DOI and the license). See: https://creativecommons.org/licenses/by-nc-nd/4.0/.

\section{References}

1. Hirano S, Tanaka E, Tsuchikawa T, et al. Techniques of biliary reconstruction following bile duct resection (with video). J Hepatobiliary Pancreat Sci 2012;19:203-9.

2. Hori T, Aisu Y, Yamamoto M, et al. Laparoscopic approach for choledochojejunostomy. Hepatobiliary Pancreat Dis Int 2019;18:285-8.

3. Singh DP, Arora S. Evaluation of biliary enteric anastomosis in benign biliary disorders. Indian J Surg 2014;76:199-203.

4. Bray F, Ferlay J, Soerjomataram I, et al. Global cancer statistics 2018: GLOBOCAN estimates of incidence and mortality worldwide for 36 cancers in 185 countries. CA Cancer J Clin 2018;68:394-424.

5. Chan CHY. Taming the BEASt: Management of Biliary-Enteric Anastomotic Strictures. Dig Dis Sci 2019;64:3363-5.

6. Kadaba RS, Bowers KA, Khorsandi S, et al. Complications of biliary-enteric anastomoses. Ann R Coll Surg Engl 2017;99:210-5.

7. Bettschart V, Clayton RA, Parks RW, et al. Cholangiocarcinoma arising after biliary-enteric drainage procedures for benign disease. Gut 2002;51:128-9.

8. Yang JR, Zhou J, Wang L, et al. A modified technique of biliary-enteric anastomosis with two-point interrupted eversion suture on the posterior wall: A surgeon's experience. Asian J Surg 2019;42:580-1.

9. Okabayashi T, Shima Y, Sumiyoshi T, et al. Incidence and Risk Factors of Cholangitis after Hepaticojejunostomy. J Gastrointest Surg 2018;22:676-83.

10. Booij KAC, Coelen RJ, de Reuver PR, et al. Longterm follow-up and risk factors for strictures after hepaticojejunostomy for bile duct injury: An analysis of surgical and percutaneous treatment in a tertiary center. Surgery 2018;163:1121-7.

11. Birgin E, Téoule P, Galata C, et al. Cholangitis following biliary-enteric anastomosis: A systematic review and metaanalysis. Pancreatology 2020;20:736-45.

12. Nakai Y. Treatment of Long-Limb Biliary-Enteric Anastomotic Strictures: ERCP, PTBD, or EUS? Dig Dis Sci 2019;64:2379-80.

13. A European-African HepatoPancreatoBiliary Association (E-AHPBA) Research Collaborative Study management group; Other members of the EuropeanAfrican HepatoPancreatoBiliary Association Research Collaborative. Post cholecystectomy bile duct injury: early, intermediate or late repair with hepaticojejunostomy - an E-AHPBA multi-center study. HPB (Oxford) 2019;21:1641-7.

14. Felder SI, Menon VG, Nissen NN, et al. Hepaticojejunostomy using short-limb Roux-en-Y reconstruction. JAMA Surg 2013;148:253-7.

15. Xu M, Wang M, Zhu F, et al. A New Approach for Rouxen-Y Reconstruction After Pancreaticoduodenectomy. Hepatobiliary Pancreat Dis Int 2014;13:649-53.

16. Cuendis-Velázquez A, Bada-Yllán O, Trejo-Ávila M, et al. Robotic-assisted Roux-en-Y hepaticojejunostomy after bile duct injury. Langenbecks Arch Surg 2018;403:53-9.

17. Sandhu J, Swersky A, Salsamendi J, et al. Utilization of a Modified Roux-en-Y Anastomosis as an Access Point for Percutaneous Transjejunal Cholangioplasty of Recurrent Biliary Strictures. Cardiovasc Intervent Radiol 2019;42:1745-50.

18. Dimou FM, Adhikari D, Mehta HB, et al. Incidence of hepaticojejunostomy stricture after hepaticojejunostomy. Surgery 2016;160:691-8.

19. Asano T, Natsume S, Senda $Y$, et al. Incidence and risk factors for anastomotic stenosis of continuous hepaticojejunostomy after pancreaticoduodenectomy. J Hepatobiliary Pancreat Sci 2016;23:628-35.

20. Narayanan SK, Chen Y, Narasimhan KL, et al. Hepaticoduodenostomy versus hepaticojejunostomy after resection of choledochal cyst: a systematic review and meta-analysis. J Pediatr Surg 2013;48:2336-42.

21. Fan C, Zhang H, Yan X, et al. Advanced Roux-en-Y hepaticojejunostomy with magnetic compressive anastomats in obstructive jaundice dog models. Surg Endosc 2018;32:779-89.

22. Laursen HB, Thorsøe HJ, Funch-Jensen P, et al. Roboticassisted laparoscopic Roux-en-Y choledochojejunostomy in pigs. J Hepatobiliary Pancreat Surg 2005;12:167-72.

23. Zhang XQ, Tian YH, Xu Z, et al. An end-to-end anastomosis model of guinea pig bile duct:A6-mo observation. World J Gastroenterol 2011;17:789-95.

24. Lee JS, Hong TH. In vivo porcine training model for laparoscopic Roux-en-Y choledochojejunostomy. Ann Surg Treat Res 2015;88:306-10.

25. de Aro Braz MJ, Corbi LE, Tannuri ACA, et al. Analysis of the reversibility of biliary cirrhosis in young rats submitted to biliary obstruction. J Pediatr Surg 2018;53:1408-13.

26. Corbi LE, Tannuri ACA, de Aro Braz MJ, et al. 
Does Biliodigestive Anastomosis Have Any Effect on the Reversal of Hepatopulmonary Syndrome in a Biliary Cirrhosis Experimental Model? Dig Dis Sci 2019;64:3192-202.

27. Brunner M, Stockheim J, Krautz C, et al. Continuous or Interrupted Suture Technique for Hepaticojejunostomy? A National Survey. BMC Surg 2018;18:84.

28. Kerwin LY, El TalAK, Stiff MA, et al. Scar prevention and remodeling: a review of the medical, surgical, topical and light treatment approaches. Int J Dermatol 2014;53:922-36.

29. Larson-Casey JL, CarterAB. Assay to evaluate BAL Fluid regulation of Fibroblast $\alpha$-SMA Expression. Bio Protoc 2016;6:e2009.

30. Yen YH, Pu CM, Liu CW, et al. Curcumin accelerates cutaneous wound healing via multiple biological actions: The involvement of TNF- $\alpha$, MMP-9, $\alpha$-SMA, and collagen. Int Wound J 2018;15:605-17.

31. Zhang X, Tian Y, Xu Z, et al. Healing process of the guinea pig common bile duct after end-to-end anastomosis: pathological evaluation after 6 months. Eur Surg Res 2011;46:194-206.

32. Ling E, Robinson DS. Transforming growth factor-beta1: its anti-inflammatory and pro-fibrotic effects. Clin Exp Allergy 2002;32:175-8.

33. Kim KK, Sheppard D, Chapman HA. TGF- $\beta 1$ Signaling and Tissue Fibrosis. Cold Spring Harb Perspect Biol 2018;10:a022293.

Cite this article as: Lyu SC, Wang J, Zhou L, Zhu JQ, Pan F, Jiang T, Lang R, He Q. Mechanism of scar formation following Roux-en-Y choledochojejunostomy in a novel rat model of obstructive jaundice. Ann Transl Med 2021;9(6):456. doi: 10.21037/atm-20-5135
34. Sapudom J, Wu X, Chkolnikov M, et al. Fibroblast fate regulation by time dependent TGF- $\beta 1$ and IL-10 stimulation in biomimetic $3 \mathrm{D}$ matrices. Biomater Sci 2017;5:1858-67.

35. Greaves NS, Ashcroft KJ, Baguneid M, et al. Current understanding of molecular and cellular mechanisms in fibroplasia and angiogenesis during acute wound healing. J Dermatol Sci 2013;72:206-17.

36. Xu Y, Sun X, Zhang R, et al. A Positive Feedback Loop of TET3 and TGF- $\beta 1$ Promotes Liver Fibrosis. Cell Rep 2020;30:1310-1318.e5.

37. Siqueira OHK, Oliveira KJ, Carvalho ACG, et al. Effect of tamoxifen on fibrosis, collagen content and transforming growth factor- $\beta 1,-\beta 2$ and $-\beta 3$ expression in common bile duct anastomosis of pigs. Int J Exp Pathol 2017;98:269-77.

38. Block L, Gosain A, King TW. Emerging Therapies for Scar Prevention. Adv Wound Care (New Rochelle) 2015;4:607-14.

39. Xu J, Geng ZM, Ma QY. Microstructural and ultrastructural changes in the healing process of bile duct trauma. Hepatobiliary Pancreat Dis Int 2003;2:295-9.

40. Siqueira OH, Herani Filho B, Paula RE, et al. Tamoxifen decreases the myofibroblast count in the healing bile duct tissue of pigs. Clinics (Sao Paulo) 2013;68:101-6.

41. Yang Q, Wang J, Liu F, et al. A Novel Rabbit Model for Benign Biliary Stricture Formation and the Effects of Medication Infusions on Stricture Formation. Dig Dis Sci 2018;63:2653-61. 